HEPATOLOGY

\title{
Expression of the caudal-type homeodomain transcription factor CDX2 is related to clinical outcome in biliary tract carcinoma
}

\author{
Yu-Ting Chang, ${ }^{*}$ Chiun Hsu, ${ }^{\dagger}$ Yung-Ming Jeng ${ }^{\ddagger}$ Ming-Chu Chang, ${ }^{*}$ Shu-Chen Wei* and Jau-Min Wong* \\ Departments of *Internal Medicine, ${ }^{\dagger}$ Oncology, and ${ }^{\ddagger}$ Pathology, National Taiwan University Hospital, Taipei, Taiwan
}

\author{
Key words \\ bile duct carcinoma, biliary tract carcinoma, \\ CDX2, survival.
}

Accepted for publication 19 February 2006.

\section{Correspondence}

Dr Jau-Min Wong, Department of Internal

Medicine, National Taiwan University Hospital, No. 7 Chung Shan South Road, Taipei, Taiwan. Email: jmwong@ha.mc.ntu.edu.tw

\begin{abstract}
Background and Aim: The caudal-type homeodomain transcriptional factor CDX2, a member of the caudal-related homeobox gene family, plays a crucial role in the regulation of cell proliferation and differentiation in the gut. Recent studies have reported that expression of CDX2 was an independent marker of outcome in patients with resected adenocarcinoma of ampulla of Vater, gastric cancer, and colon cancer. The clinicopathological significance of CDX2 expression has hitherto remained unclear in biliary tract carcinoma (BTC). The aim of this study was to determine whether CDX2 expression in BTC indicates clinical outcome.

Methods: The expression of CDX2 was investigated immunohistochemically in surgically resected specimens from 164 patients with BTC, including 74 intrahepatic cholangiocarcinomas, 49 extrahepatic cholangiocarcinomas, and 41 gallbladder carcinomas. The correlation between expression of CDX2 and clinicopathological factors, including overall survival, tumor location, tumor stage, and degree of tumor differentiation, was examined in patients with BTC.

Results: In total, 27 of the $164(16.46 \%)$ patients with BTC expressed CDX2. The frequency of CDX2 expression was much higher in the extrahepatic cholangiocarcinomas $(22.45 \%)$ and gallbladder carcinomas $(29.27 \%)$ than in the intrahepatic cholangiocarcinomas $(5.41 \%)$, the difference being statistically significant $(P=0.002)$. Factors influencing survival on univariate analysis were tumor stage $(P<0.00001)$, degree of tumor differentiation $(P=0.0002)$, and $\mathrm{CDX} 2$ expression $(P=0.01)$. On multivariate analysis using the Cox proportional hazard model, $\mathrm{CDX} 2$ expression and tumor stage were independent prognostic factors in patients with BTC.

Conclusion: Expression of CDX2 was an independent indicator of outcome in patients with BTC in addition to tumor stage and tumor differentiation. The mechanism by which CDX2 expression influences behavior of biliary tract carcinoma needs further investigation.
\end{abstract}

\section{Introduction}

The biliary tract consists of an interconnected system of intra- and extrahepatic ducts that transport bile secreted from the liver to the digestive tract. Biliary tract carcinomas (BTCs), which include cancers of the gallbladder and intra- and extrahepatic biliary trees, are relatively infrequent but highly lethal diseases that are notoriously difficult to diagnose and treat, and have incidences that are steadily increasing worldwide. ${ }^{1-3}$ Diagnosis of BTC usually occurs at an advanced stage of the disease, especially intrahepatic cholangiocarcinomas and gallbladder cancer. In general, the prognosis for patients with advanced BTC at any site is dismal. ${ }^{4}$ Local invasion, extensive regional lymph node metastasis, distant metastases, and vascular encasement and invasion often preclude resection, and neither radiation nor conventional chemotherapy significantly improves survival or quality of life. Understanding the pathogenesis and clinicopathological characteristics of BTC might help to improve the overall treatment strategy for the tumor.

The caudal-type homeodomain transcriptional factor CDX2, a member of the caudal-related homeobox gene family, is mostly expressed in the villi or differentiated cell component of the intestine. ${ }^{5} \mathrm{CDX} 2$ plays a crucial role in the regulation of cell proliferation and differentiation in the gut. ${ }^{6,7}$ Ectopic CDX2 expression has been seen in intestinal metaplasia of stomach ${ }^{8-11}$ and Barrett's 
esophagus. ${ }^{12}$ CDX2 is also expressed in gastric carcinoma, ${ }^{13}$ cholangioarcinoma ${ }^{14}$ and esophageal carcinoma. ${ }^{15}$ A previous study has shown that expression of CDX2 is an independent marker of outcome in patients with resected adenocarcinoma of ampulla of Vater, which is composed of three distinct epithelial elements including duodenal mucosa, pancreatic ductal epithelium and biliary ductal epithelium. ${ }^{16}$ Those tumors with markers of intestinal epithelium as compared with biliary epithelium have better prognosis. ${ }^{16}$ In addition, loss of CDX2 expression has been associated with a poor prognosis in colon cancer ${ }^{17}$ and gastric cancer. ${ }^{13}$ The clinicopathological significance of the phenotype and CDX2 expression has hitherto remained unclear in BTC. In the present study, we therefore examined the correlation between prognosis and CDX2 expression in BTC.

\section{Methods}

\section{Patient specimens}

Permission for this study was obtained from the ethics committee of National Taiwan University Hospital. Paraffin-embedded material from a series of 164 BTC surgical specimens, consisting of 74 intrahepatic cholangiocarcinomas, 49 extrahepatic cholangiocarcinomas, and 41 gallbladder carcinomas, resected at the National Taiwan University Hospital between 1995 and 2004, were used for this study.

\section{Immunohistochemistry}

Four-micrometer sections from paraffin-embedded tissues were used for immunohistochemistry. Slides were deparaffinized in fresh xylene and rehydrated through sequential graded enthanols. Antigen retrieval was performed by citrate buffer incubation (10 mmol/L, pH 6) using a microwavable pressure cooker for $20 \mathrm{~min}$. Slides were cooled for $20 \mathrm{~min}$, incubated for $5 \mathrm{~min}$ with $3 \%$ hydrogen peroxide, washed in phosphate-buffered saline (PBS) $0.1 \%$ Triton X-100 (pH 7.6), blocked for $20 \mathrm{~min}$ in $20 \%$ normal bovine serum, and incubated with mouse monoclonal CDX2 antibody (Bio Genex, San Ramon, CA, USA) diluted to 1:100 overnight at $4^{\circ} \mathrm{C}$. The next day, the slides were washed in
PBS $0.1 \%$ Triton $\mathrm{X}-100$ and incubated for $30 \mathrm{~min}$ in a 1:200 dilution of biotinylated antimouse secondary antibody. The DakoCytomation LSAB2 System-HRP (DakoCytomation, Carpentaria, CA, USA) was used to visualize antibody binding, and slides were subsequently counterstained with hematoxylin.

\section{Immunolabeling analysis and scoring}

Slides were examined by an independent pathologist (Y.-M.J.). The labeling index criterion was set at less than $25 \%$ versus more than $25 \%$ positively labeled cancer cells.

\section{Statistical analysis and patient outcomes}

The association of CDX2 expression among clinicopathological factors was studied by $\chi^{2}$ analysis of proportions, Fisher's exact test, and Student's $t$-test when appropriate. Kaplan-Meier survival analysis and log-rank tests were performed for univariate survival analysis, and the Cox proportional hazards model for multivariate survival analysis. $P<0.05$ was accepted as statistically significant. The variables assessed were patient age at diagnosis, sex, tumor location, tumor stage, degree of tumor differentiation, and CDX2 expression. The statistical calculations were carried out using StatView-J 5.0 statistical software (SAS Institute, Cary, NC, USA).

\section{Results}

\section{CDX2 expression in bile duct carcinomas}

The study included 164 patients with mean age of 63.7 years (range, 30-95 years); 52\% of patients were female. The mean follow-up period was 21.9 months (range $0.5-117.3$ months). The clinicopathological features and the expression of CDX2 in 164 patients with biliary tract carcinoma are shown in Table 1 . In total, 27 of the $164(16.46 \%)$ BTCs expressed CDX2, in which positive CDX2 staining was seen in the nuclei of carcinoma cells (Fig. 1). When we compared CDX2 expression with the clinicopathological factors in the BTCs, there was no statistically significant difference in patient sex or age, and tumor grade or stage between the CDX2-positive and CDX2-negative groups (Table 2). However,

Table 1 Clinicopathological features of 164 patients with biliary tract carcinoma

\begin{tabular}{|c|c|c|c|c|}
\hline Characteristic & $\begin{array}{l}\text { Intrahepatic bile duct } \\
\text { carcinoma }(n=74)\end{array}$ & $\begin{array}{l}\text { Extrahepatic bile duct } \\
\text { carcinoma }(n=49)\end{array}$ & $\begin{array}{l}\text { Gallbladder carcinoma } \\
(n=41)\end{array}$ & $\begin{array}{l}\text { Total } \\
(n=164)\end{array}$ \\
\hline \multicolumn{5}{|l|}{ Age (years) } \\
\hline Mean \pm SD & $61.3 \pm 11.7$ & $63.5 \pm 11.3$ & $68.5 \pm 13.1$ & $63.7 \pm 12.2$ \\
\hline Range & $30-87$ & $39-82$ & $41-95$ & $30-95$ \\
\hline \multicolumn{5}{|l|}{ Sex } \\
\hline Male & 35 & 29 & 14 & 78 \\
\hline Female & 39 & 20 & 27 & 86 \\
\hline \multicolumn{5}{|l|}{ Tumor grade } \\
\hline Well differentiated & 18 & 18 & 13 & 49 \\
\hline Moderately differentiated & 38 & 26 & 22 & 86 \\
\hline Poorly differentiated & 18 & 5 & 6 & 29 \\
\hline \multicolumn{5}{|l|}{ Stage } \\
\hline I and II & 40 & 17 & 10 & 67 \\
\hline III and IV & 34 & 32 & 31 & 97 \\
\hline
\end{tabular}



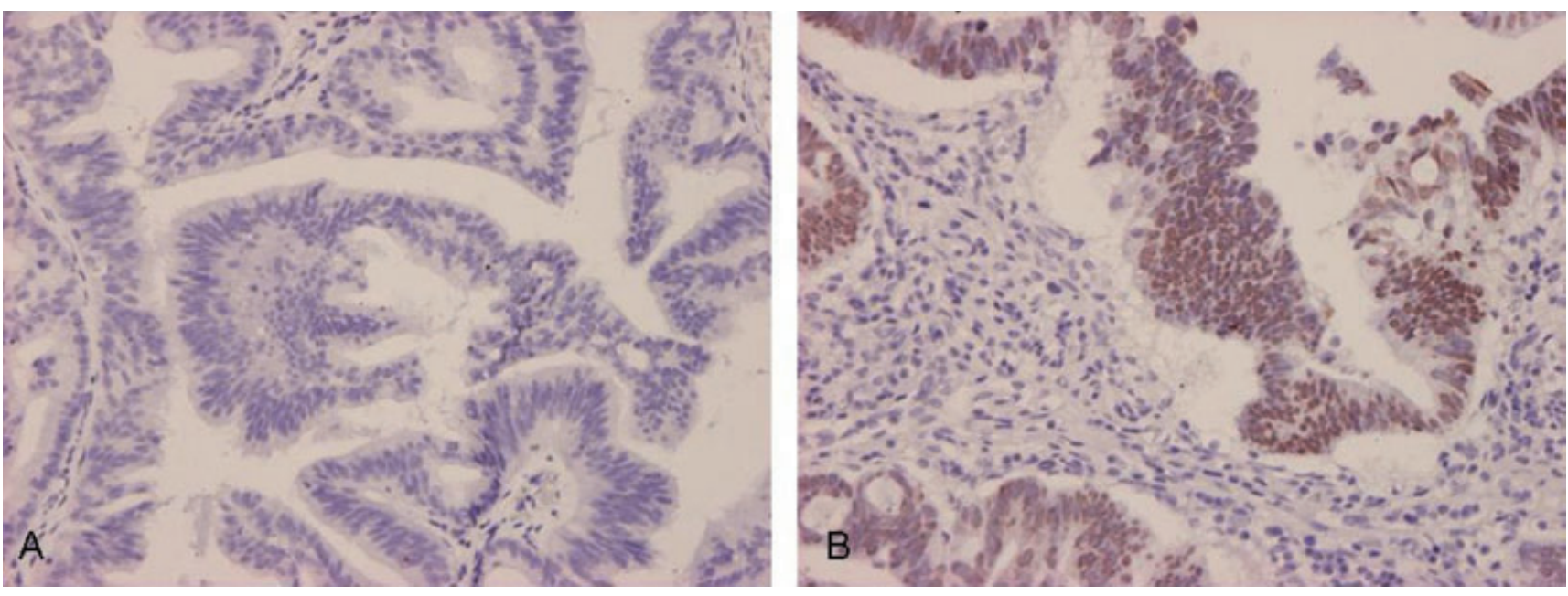

Figure 1 Expression of CDX2 in extrahepatic cholangiocarcinoma: (a) negative expression; (b) positive expression in the nuclei of carcinoma cells (original magnification $\times 200$ ).

Table 2 Comparison of CDX2 expression and the clinicopathological factors in biliary tract carcinoma

\begin{tabular}{llll}
\hline Characteristic & CDX2 (+) & CDX2 (-) & $P$-value \\
\hline Age (years) & $65.5 \pm 12.6$ & $63.4 \pm 12.2$ & 0.41 \\
Sex & & & \\
$\quad$ Male & 14 & 64 & 0.68 \\
$\quad$ Female & 13 & 73 & \\
Tumor grade & & & \\
$\quad$ Well differentiated & 11 & 38 & 0.07 \\
$\quad$ Moderately differentiated & 15 & 71 & \\
$\quad$ Poorly differentiated & 1 & 28 & \\
Stage & & & \\
$\quad$ I and II & 11 & 56 & 1 \\
III and IV & 16 & 81 & \\
Location & & & \\
Intrahepatic & 4 & 70 & \\
$\quad$ Extrahepatic & 11 & 38 & \\
$\quad$ Gallbladder & 12 & 29 & \\
\hline
\end{tabular}

the frequency of CDX2 expression was much higher in the extrahepatic cholangiocarcinomas $(22.45 \%)$ and gallbladder carcinomas $(29.27 \%)$ than in the intrahepatic cholangiocarcinomas $(5.41 \%)$, the difference being statistically significant $(P=0.002)$ (Table 2).

\section{CDX2 expression is associated with better prognosis in patients with BTC}

Factors influencing survival on univariate analysis were tumor stage (mean survival 54.2 months, 95\% confidence interval [CI] 41.3-67.0 months for stage I-II vs 19.9 months, 95\% CI 14.1-25.7 months for stage III-IV; $P<0.00001$ ), degree of tumor differentiation (mean survival 42.8 months, 95\% CI 30.1-55.4 months for well-differentiated carcinoma vs 34.7 months, $95 \%$ CI 25.3-44.1 months for moderately differentiated carcinoma $v s$
10.8 months, 95\% CI 6.7-14.9 months for poorly differentiated carcinoma; $P=0.0002$ ), and CDX2 expression (mean survival 51.5 months, 95\% CI 33.8-69.2 months for CDX2-positive tumors vs 29.2 months, 95\% CI 22.2-36.2 months for CDX2negative tumors; $P=0.01)$. Patient age at diagnosis, sex, and tumor location did not influence survival. The results of univariate analysis are shown in Table 3 and Kaplan-Meier survival curves are shown in Fig. 2.

On multivariate analysis using the Cox proportional hazard model, CDX2 expression and tumor stage were independent prognostic factors in patients with BTC (Table 4). Multivariateadjusted hazard ratio of death for CDX2 in various subsets of patients with BTC showed that CDX2 expression was associated with a significant survival advantage in female patients, patients with advanced stage disease, and patients with well-differentiated carcinoma (Table 5).

\section{Discussion}

CDX2 expression is intestine-specific in the embryo and during adulthood. CDX2 is expressed in the nuclei of epithelial cells throughout the intestine, from duodenum to rectum. CDX2 expression has been observed in intestinal metaplasia and adenocarcinoma of stomach but not in normal gastric mucosa. ${ }^{8}$ In previous studies, CDX2 expression was not detected in normal gallbladder mucosa and epithelium of bile duct. ${ }^{16,18}$ We found that $5.4 \%$ of intrahepatic cholangiocarcinomas, $22.5 \%$ of extrahepatic biliary tract carcinomas, and $29.3 \%$ gallbladder carcinomas expressed CDX2 in our study. In addition, CDX2 expression alone was an independent predictor of survival after resection of BTC. Advanced tumor stage and poor differentiation of tumor were associated with a poor prognosis of BTC.

In our study, there was a trend of increasing expression of CDX2 in BTCs that were located nearer the intestine. There was no differential expression of CDX2 in BTC according to different age groups, sex, grades of tumor differentiation, and tumor stages. Although the gallbladder and bile ducts are from the same embryologic origin, ${ }^{19}$ the intrahepatic cholangiocarcinomas, extrahepatic 
Table 3 Clinicopathological features and survival data in 164 patients with biliary tract carcinoma

\begin{tabular}{|c|c|c|c|c|}
\hline Characteristic & No. patients & Mean survival (months, 95\% Cl) & $P($ log-rank $)$ & Crude hazard ratio of death $(95 \% \mathrm{Cl})$ \\
\hline \multicolumn{5}{|l|}{ Sex } \\
\hline Male & 78 & $32.7(23.7-41.6)$ & & 1 \\
\hline Female & 86 & $33.6(23.9-43.3)$ & 0.62 & $1.09(0.77-1.57)$ \\
\hline \multicolumn{5}{|l|}{ Age } \\
\hline$<65$ years & 83 & $37.2(27.1-47.3)$ & & \\
\hline$\geq 65$ years & 81 & $29.0(21.0-37.1)$ & 0.22 & $1.25(0.88-1.80)$ \\
\hline \multicolumn{5}{|l|}{ Stage } \\
\hline | and || & 67 & $54.2(41.3-67.0)$ & & 1 \\
\hline III and IV & 97 & $19.9(14.1-25.7)$ & $<0.0001$ & $2.73(1.84-4.05)^{* *}$ \\
\hline \multicolumn{5}{|l|}{ Differentiation } \\
\hline Well & 49 & $42.8(30.1-55.3)$ & & 1 \\
\hline Moderate & 86 & $34.7(25.3-44.1)$ & & $1.24(0.81-1.90)$ \\
\hline Poor & 29 & $10.8(6.7-14.9)$ & 0.0002 & $1.86(0.90-3.84)^{* *}$ \\
\hline \multicolumn{5}{|l|}{ Location } \\
\hline Intrahepatic & 74 & $32.3(22.5-42.2)$ & & 1 \\
\hline Extrahepatic & 49 & $40.3(27.6-52.9)$ & & $0.80(0.51-1.23)$ \\
\hline Gallbladder & 41 & $28.5(16.1-40.9)$ & 0.20 & $1.24(0.80-1.91)$ \\
\hline \multicolumn{5}{|c|}{ CDX2 expression } \\
\hline Positive & 27 & $51.5(33.8-69.2)$ & 0.01 & $0.51(0.29-0.88)^{*}$ \\
\hline Negative & 137 & $29.2(22.2-36.2)$ & & 1 \\
\hline
\end{tabular}

${ }^{*} P<0.05 ;{ }^{*} P<0.005$.

A

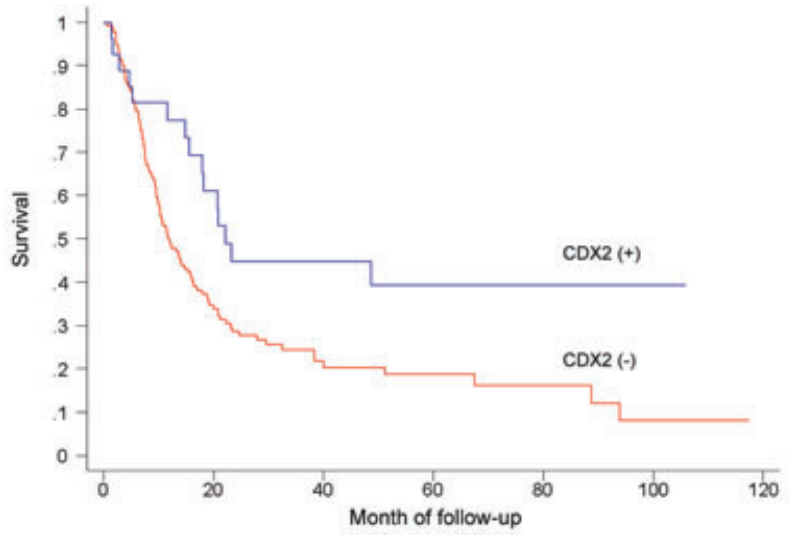

C

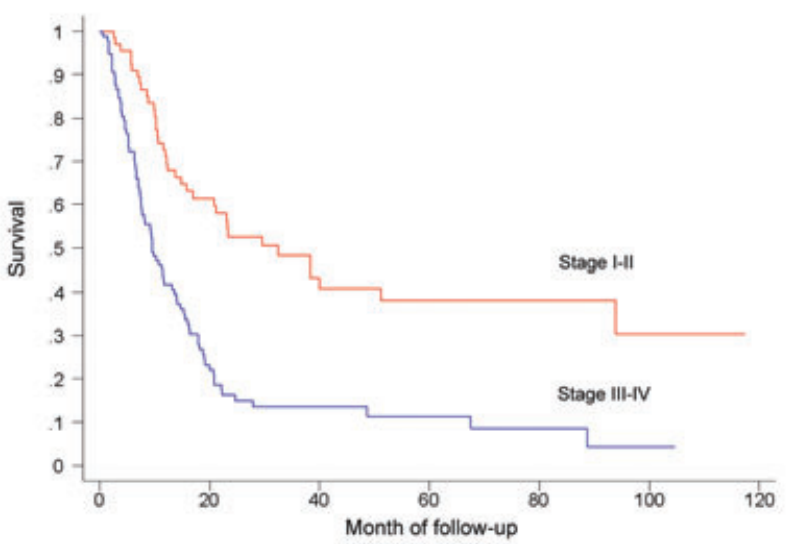

B

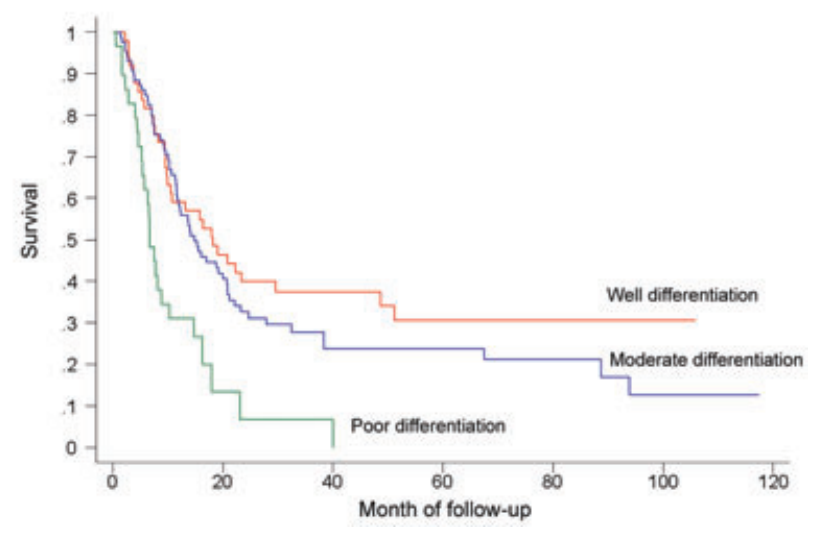

D

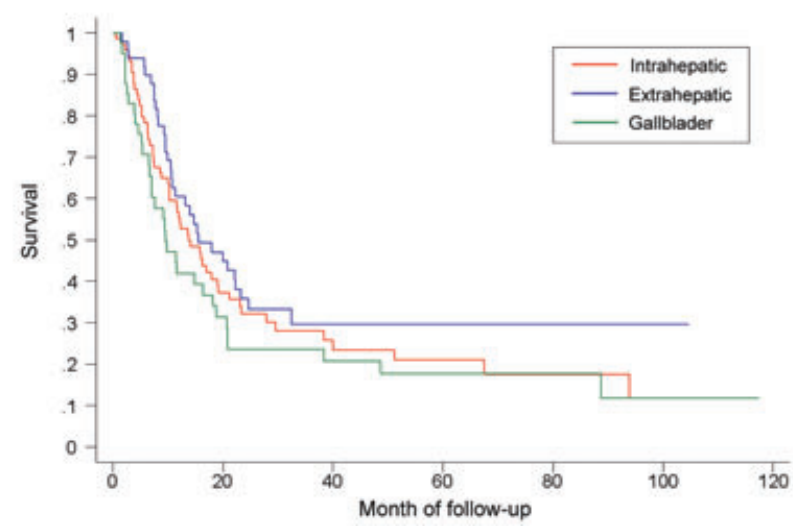

Figure 2 Kaplan-Meier survival curves for clinicopathological factors and CDX2 expression in biliary tract carcinomas according to: (a) CDX2 expression; (b) tumor differentiation; (c) tumor stage; and (d) tumor location. 
Table 4 Multivariate-adjusted hazard ratio of death in 164 patients with biliary tract carcinoma

\begin{tabular}{ll}
\hline Characteristic & Adjusted hazard ratio of death $(95 \% \mathrm{Cl})$ \\
\hline Sex & 1 \\
Male & $0.96(0.65-1.40)$ \\
Female & \\
Age & 1 \\
$\quad<65$ years & $1.20(0.82-1.76)$ \\
$\geq 65$ years & \\
Stage & 1 \\
I and II & $3.08(2.01-4.73)^{* *}$ \\
III and IV & \\
Differentiation & 1 \\
Well & $1.09(0.70-1.69)$ \\
Moderate & $1.93(0.92-4.05)$ \\
Poor & 1 \\
Location & 1 \\
Intrahepatic & $0.68(0.42-1.10)$ \\
Extrahepatic & $10.76-1.97)$ \\
Gallbladder & \\
CDX2 expression & \\
Positive & \\
Negative & \\
\hline$* P<0.05 * * * 27-0.86)^{*}$ \\
\hline
\end{tabular}

${ }^{*} P<0.05 ;{ }^{*} P<0.005$.

Table 5 Multivariate-adjusted hazard ratio of death for CDX2 in various subsets of patients with biliary tract carcinomas

\begin{tabular}{ll}
\hline Characteristic & Adjusted hazard ratio of death for CDX2 $(95 \% \mathrm{CI})^{\dagger}$ \\
\hline $\begin{array}{l}\text { Sex } \\
\text { Male }\end{array}$ & $0.51(0.23-1.17)$ \\
Female & $0.35(0.15-0.83)^{*}$ \\
Age & \\
$\quad<65$ years & $0.53(0.21-1.31)$ \\
$\geq 65$ years & $0.49(0.23-1.06)$ \\
Stage & \\
I and II & $0.48(0.16-1.44)$ \\
III and IV & $0.45(0.22-0.90)^{*}$ \\
Differentiation & \\
Well & $0.29(0.10-0.86)^{*}$ \\
Moderate & $0.72(0.33-1.54)$ \\
Poor & $3.14(0.32-30.79)$ \\
Location & \\
Intrahepatic & $0.62(0.14-2.84)$ \\
Extrahepatic & $0.40(0.15-1.09)$ \\
Gallbladder & $0.50(0.20-1.27)$
\end{tabular}

${ }^{*} P<0.05$. ${ }^{\dagger}$ Adjustment for sex, age stage, differentiation and location except for the stratifying variables.

cholangiocarcinomas, and gallbladder carcinomas have different clinical manifestations. ${ }^{4}$ Differential expression of CDX2 might partially explain the different clinical manifestations in BTC. Our results suggest that BTC with markers of intestinal differentiation may have a better prognosis.
CDX2 is known to be one of the good prognostic markers in patients with gastric carcinoma, ${ }^{13,20}$ colon carcinoma, ${ }^{17}$ pancreatic tumor, ${ }^{21}$ and carcinoma of ampulla of Vater. ${ }^{16}$ In a previous study, CDX2 expression was observed in both dysplasia and intramucosal tumor of gallbladder carcinoma and was related to histological differentiation and MUC2 expression, suggesting that CDX2 expression is an early event in gallbladder carcinogenesis. ${ }^{18}$ However, the role of CDX2 as a prognostic marker was not clear in that study. ${ }^{18}$ Hong et al. have shown that CDX2 was expressed in $37.3 \%$ of extrahepatic BTCs and more frequently in tumors with papillary growth and no vascular invasion. ${ }^{22}$ In addition, patients with both CDX2 and MUC2 expressing extrahepatic BTC had a better overall survival in univariate but not multivariate analysis than patients with other tumors. ${ }^{22}$ In our study, the expression of CDX2 was associated with a significant survival advantage in patients with BTC by univariate and multivariate analysis. The results suggest that mechanisms apart from tumor stage and differentiation are influencing the outcome in patients with CDX2positive tumors. Previous studies have shown the role of CDX2 in promoting a more differentiated cellular phenotype ${ }^{6}$ and in the regulation of cell adhesion. ${ }^{23}$ Forced expression of CDX2 in epithelial cell lines inhibits cell proliferation and stimulates cell differentiation and apoptosis. ${ }^{17,24,25} \mathrm{CDX} 2$-dependent regulation of cell proliferation may be an important factor in defining the prognosis of the patients with BTC.

A survival advantage was observed in subgroups of patients with BTC with expression of CDX2 in our study, including female patients, patients with advanced stage disease, and patients with well-differentiated tumors. It is not difficult to understand why patients with advanced stage disease and patients with welldifferentiated tumors have an advantage with regard to survival due to the role of CDX2 in cell differentiation and proliferation. The reasons why female patients with CDX2 expression have a survival advantage are not clear. There have been no studies to delineate the relationship between CDX2 expression and the effect of estrogen or other hormones. The role of CDX2 in female patients with BTC needs further investigation.

In summary, expression of CDX2 influenced the overall survival in patients with BTC. CDX2 expression was an independent marker of outcome in addition to tumor stage and tumor differentiation in patients with BTC. The mechanism by which CDX2 expression influences behavior of biliary tract carcinoma needs further investigation.

\section{References}

1 Shaib Y, El-Serag HB. The epidemiology of cholangiocarcinoma. Semin. Liver Dis. 2004; 24: 115-25.

2 Olnes MJ, Erlich R. A review and update on cholangiocarcinoma. Oncology 2004; 66: 167-79.

3 Wistuba II, Gazdar AF. Gallbladder cancer: lessons from a rare tumour. Nat. Rev. Cancer 2004; 4: 695-706.

4 de Groen PC, Gores GJ, LaRusso NF et al. Biliary tract cancers. $N$. Engl. J. Med. 1999; 341: 1368-78.

5 Silberg DG, Swain GP, Suh ER et al. Cdx1 and cdx2 expression during intestinal development. Gastroenterology 2000; 119: 961-71.

6 Suh E, Traber PG. An intestine-specific homeobox gene regulates proliferation and differentiation. Mol. Cell. Biol. 1996; 16: 619-25.

7 Lorentz O, Duluc I, Arcangelis AD et al. Key role of the Cdx2 
homeobox gene in extracellular matrix-mediated intestinal cell differentiation. J. Cell Biol. 1997; 139: 1553-65.

8 Bai YQ, Yamamoto H, Akiyama Y et al. Ectopic expression of homeodomain protein CDX2 in intestinal metaplasia and carcinomas of the stomach. Cancer Lett. 2002; 176: 47-55.

9 Almeida R, Silva E, Santos-Silva F et al. Expression of intestinespecific transcription factors, CDX1 and CDX2, in intestinal metaplasia and gastric carcinomas. J. Pathol. 2003; 199: 36-40.

10 Silberg DG, Sullivan J, Kang E et al. Cdx2 ectopic expression induces gastric intestinal metaplasia in transgenic mice. Gastroenterology 2002; 122: 689-96.

11 Mutoh H, Hakamata Y, Sato K et al. Conversion of gastric mucosa to intestinal metaplasia in Cdx2-expressing transgenic mice. Biochem. Biophys. Res. Commun. 2002; 294: 470-9.

12 Eda A, Osawa H, Satoh K et al. Aberrant expression of CDX2 in Barrett's epithelium and inflammatory esophageal mucosa. J. Gastroenterol. 2003; 38: 14-22.

13 Mizoshita T, Tsukamoto T, Nakanishi $\mathrm{H}$ et al. Expression of Cdx2 and the phenotype of advanced gastric cancers: relationship with prognosis. J. Cancer Res. Clin. Oncol. 2003; 129: 727-34.

14 Ishikawa A, Sasaki M, Ohira S et al. Aberrant expression of CDX2 is closely related to the intestinal metaplasia and MUC2 expression in intraductal papillary neoplasm of the liver in hepatolithiasis. Lab. Invest. 2004; 84: 629-38.

15 Phillips RW, Frierson HF Jr, Moskaluk CA. Cdx2 as a marker of epithelial intestinal differentiation in the esophagus. Am. J. Surg. Pathol. 2003; 27: 1442-7.

16 Hansel DE, Maitra A, Lin JW et al. Expression of the caudal-type homeodomain transcription factors CDX 1/2 and outcome in carcinomas of the ampulla of Vater. J. Clin. Oncol. 2005; 23: 1811-18.

17 Mallo GV, Soubeyran P, Lissitzky JC et al. Expression of the Cdx1 and Cdx2 homeotic genes leads to reduced malignancy in colon cancer-derived cells. J. Biol. Chem. 1998; 273: 14030-6.

$18 \mathrm{Wu}$ XS, Akiyama Y, Igari T et al. Expression of homeodomain protein CDX2 in gallbladder carcinomas. J. Cancer Res. Clin. Oncol. 2005; 131: 271-8.

19 Sergi C, Adam S, Kahl P et al. The remodeling of the primitive human biliary system. Early Hum. Dev. 2000; 58: 167-78.

20 Seno H, Oshima M, Taniguchi MA et al. CDX2 expression in the stomach with intestinal metaplasia and intestinal-type cancer: prognostic implications. Int. J. Oncol. 2002; 21: 769-74.

21 Matsumoto K, Mizoshita T, Tsukamoto T et al. Cdx2 expression in pancreatic tumors: relationship with prognosis of invasive ductal carcinomas. Oncol. Rep. 2004; 12: 1239-43.

22 Hong SM, Cho H, Moskaluk CA et al. CDX2 and MUC2 protein expression in extrahepatic bile duct carcinoma. Am. J. Clin. Pathol. 2005; 124: 361-70.

23 Hinoi T, Lucas PC, Kuick R et al. CDX2 regulates liver intestinecadherin expression in normal and malignant colon epithelium and intestinal metaplasia. Gastroenterology 2002; 123: 1565-77.

24 Hinoi T, Loda M, Fearon ER. Silencing of CDX2 expression in colon cancer via a dominant repression pathway. J. Biol. Chem. 2003; 278: 44608-16.

25 Lorentz O, Cadoret A, Duluc I et al. Downregulation of the colon tumour-suppressor homeobox gene $\mathrm{Cdx}-2$ by oncogenic ras. Oncogene 1999; 18: 87-92. 